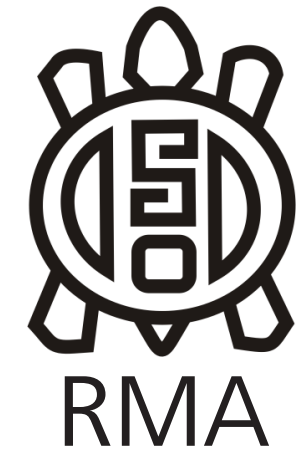

Antropología Social

\section{Los mecanismos de innovación léxica y la reconfiguración de la modernidad indígena en guaraní chaqueño}

\author{
The mechanisms of lexical innovation and the reconfiguration of \\ indigenous modernity in Chacoan Guarani
}

María Agustina Morando*

*IICS/CONICET-UCA, Argentina. E-mail: agustinamorando@uca.edu.ar

\title{
Resumen
}

El objetivo de este trabajo es documentar los mecanismos de innovación léxica en la lengua guaraní chaqueña o chiriguana, que han resultado en la emergencia de una gran cantidad de palabras nuevas. El foco estará puesto, en particular, en tres modos en los que pueden manifestarse estos procesos: mediante la creación de nuevas palabras, la incorporación de préstamos, y mediante las operaciones de extensión semántica a partir de las cuales palabras ya existentes en la lengua adquieren nuevos significados. Para ello, se trabajará tanto con materiales escritos como diccionarios, glosarios y documentos de distintos tipos en los que identifiqué elementos léxicos novedosos, como con discurso oral, obtenido a partir de mi propia investigación etnográfica. Se trata, entonces, de abordar desde una perspectiva antropológica la dinámica de los procesos de innovación léxica, recuperar los aspectos etnohistóricos que influyeron en ellos en distintos momentos y observar los modos en los que contribuyen a significar y construir la modernidad indígena.

Palabras clave: innovación léxica; Guaraní chaqueño; Lenguas indígenas; Chaco; Modernidad.

\begin{abstract}
The aim of this paper is to document the mechanisms of lexical innovation in Chacoan Guarani or Chiriguano language, which resulted in the emergence of a large number of new words. The focus is particularly on three ways in which the processes of lexical innovation can occur: the creation of new words, the incorporation of borrowings, and the operations of semantic extension whereby words already existing in the language acquire new meanings. In order to identify new lexical elements, published materials such as dictionaries, glossaries and documents of different types as well as oral discourse documented during my ethnographic research are studied. This involves approaching the dynamics of lexical innovation processes from an anthropological perspective, recovering the ethnohistorical aspects influencing them at different times and observing the ways in which they contribute to signifying and constructing indigenous modernity.
\end{abstract}

Keywords: Lexical innovation; Chacoan Guarani; Indigenous languages; Chaco; Modernity.

\section{Introducción}

Al igual que la gran mayoría de las lenguas indígenas sudamericanas, la lengua conocida en la tradición etnográfica del Chaco occidental como "guaraní chaqueño" o "chiriguano" ha atravesado en distintos momentos históricos por diversos procesos de innovación léxica que derivan en la incorporación continua de nuevas palabras al repertorio de recursos disponibles a los hablantes (Dorais, 1970; Brown y Witkowski, 1983). A partir de una mirada antropológica propongo revisar estos fenómenos, a fin de echar luz sobre los múltiples modos en los que los propios indígenas construyen verbalmente su "modernidad", es decir, cómo incorporan los elementos "no tradicionales" en respuesta a la creciente presión del frente criollo (Pitarch y Orobitg, 2012).

Pondré entonces el foco en tres modalidades en las que pueden manifestarse los procesos de innovación léxica que se produce fundamentalmente en el plano de lo discursivo, y que constituyen, por otro lado, universales translingüísticos (Casado Velarde, 2015): la creación de nuevas palabras -sobre todo por composición sobre la base de palabras ya existentes en la lengua-, la incorporación de préstamos -ya sea por adopción o imposición-, o bien, los mecanismos de extensión semántica en los cuales palabras ya existentes adquieren nuevos significados (Matoré, 1953; Monino, 1970; Haspelmath, 2008). Para ello he trabajado con diccionarios, glosarios y documentos escritos de distintos tipos y, asimismo, con material oral 
proveniente de mi trabajo de campo etnográfico entre los chanés y guaraníes del Noroeste Argentino y sudeste de Bolivia (Morando, 2015, 2020). El corpus en lengua indígena fue, además, agrupado en base a determinados dominios temáticos -sociedad civil y cotidianeidad, educación y academia, sistemas de medición y máquinasya que esto me permitirá poner en perspectiva de forma más inteligible los campos semánticos en los que estos procesos se han desarrollado.

Antes de avanzar, es preciso aclarar que el guaraní chaqueño o chiriguano pertenece a la rama meridional de la familia lingüística tupí-guaraní junto a otras lenguas como el ñandeva, el kaiowá, el mbyá o el aché. Esta lengua es hablada por cerca de 70.000 personas en el oriente boliviano, en el Noroeste Argentino y en sectores más bien dispersos del oeste de Paraguay (Dietrich, 1986, 2007; Gustafson, 2014) por una "etnia mixta" reconocida en la etnohistoria chaqueña bajo los nombres de "chiriguana", "chiriguano" o "chiriguanaes" y que, a partir de la década de 1980, se dio a conocer como "guaraní" desechando la antigua denominación por sus connotaciones despectivas (Combès, 2005; Combès y Saignes, 1995; Combès y Villar, 2007; Susnik, 1968). Este macro conjunto "chiriguano-guaraní" se compone de distintos grupos étnicos -avas, simbas, chanés, isoseños, y los llamados en ocasiones "guarayos" del oeste paraguayo- que hablan cuatro variantes dialectales homónimas, y que actualmente se autoadscriben de manera general como "guaraníes", a excepción de los chanés, que han mantenido una conciencia clara de su diferencia étnica $y$, por ende, rechazan los etnónimos "chiriguano" y "guaraní" (Bossert, Combès y Villar, 2008; Combès, 2005; Nordenskiöld, 2002 [1912]; Villar, 2006)1․

1 Los ava se encuentran en la actualidad asentados en los departamentos bolivianos de Santa Cruz, Chuquisaca y Tarija, en las provincias argentinas de Salta y Jujuy y en el departamento paraguayo de Boquerón. La presencia simba está registrada en los departamentos de Chuquisaca (provincias Hernando Siles y Luis Calvo) y de Tarija (provincia O'Connor), así como en la provincia argentina de Salta (departamento Orán) (Villar, 2006). Culturalmente cercanos a los simbas, los chanés se sitúan hoy en día en la provincia argentina de Salta (departamento Aguaray). Aunque la bibliografía postula que antiguamente pertenecían a la familia lingüística arawak, tras una serie de migraciones masivas se establecieron al oeste del Gran Chaco, sobre el piedemonte andino, donde, a partir del siglo XVI padecieron un intenso proceso de "guaranización" a manos de grupos tupí-guaraníes que también llegaban a la zona; en este sentido, los chanés poseen una importancia crucial en la formación histórica de la ya referida etnia mixta "chiriguano" (Combès y Saignes, 1995; Métraux, 1948; Susnik, 1968). Los isoseños se ubican principalmente en la provincia de Salta (departamento Aguaray) y en el valle del río Parapetí (Bolivia). Serían descendientes de una parcialidad chané que buscó refugio de la dominación guaraní en el Isoso; aunque, al ser ésta también región guaranizada, adoptaron inevitablemente la lengua guaraní (Combès, 2005; Schuchard, 1979). Una parcialidad isoseña se estableció durante la guerra del Chaco (1932-1935) en el oeste paraguayo siendo reconocida bajo el nombre de "guarayos" Esta población opta por identificarse actualmente como "guaraníes" (Riester, 2008). Al haberse utilizado dicho etnónimo en las tierras bajas con un valor genérico, no debe confundirse por tanto a este grupo con otros referidos de la misma forma como los guaraní hablantes de la provincia boliviana de Guarayos, los pausernas que habitan en la frontera de Bolivia y Brasil, o algunos grupos de habla tacana del Beni
Lo cierto es que, desde el punto de vista lingüístico, tanto la estructura gramatical como una buena parte del léxico de esta lengua se revelan muy similares en las distintas variantes dialectales habladas por esos grupos étnicos. De hecho, la "unidad dialectológica" del guaraní chaqueño o chiriguano está ampliamente fundamentada por una semejanza entre rasgos fonéticos, léxicos y gramaticales (Dietrich, 1986; Gustafson, 2014). A pesar de las singularidades étnicas y los desarrollos históricos locales que caracterizan a cada uno de los grupos étnicos que hablan esta lengua, al tomar en cuenta el contacto estrecho y fluido que mantienen recíprocamente entre sí he decidido enfocar este trabajo desde una óptica general que resulte más útil para la observación de los procesos específicos de innovación léxica.

\section{Acerca de la lengua guaraní chaqueña o chiriguana}

A continuación, describiré someramente algunos rasgos particulares de la lengua indígena que permitirán al lector un mejor acercamiento al análisis propuesto². Uno de los aspectos quizá más representativos a nivel fonológico -en contraposición con otras lenguas de la misma familia como el guaraní paraguayo, el kaiowá, el chiripá o el mbyá- es el acento llano o paroxítono, es decir, aplicado siempre en la penúltima sílaba de una palabra. Sin embargo, es importante aclarar que el mismo no es exclusivo del chiriguano, y que también puede encontrarse en otras lenguas guaraníes como el sirionó o el guarayo (Dietrich, 1986: 58). Por otra parte, la oclusión glotal, fenómeno frecuente en varias lenguas de la familia lingüística Tupí-Guaraní como el guaraní paraguayo, el mbyá o el guarayo, no se observa sino raramente en cualquiera de las variantes dialectales del guaraní chaqueño (Daviet, 2016: 49).

Desde un punto de vista tipológico, esta lengua es aglutinante-las palabras se componen de una secuencia de morfemas-y polisintética -presenta varios morfemas por palabra- (Dietrich, 1986; Gustafson, 2014). Entremos en detalle, entonces, en algunos rasgos de la morfología indispensables para comprender cómo opera la formación de palabras. Para comenzar, en lo que respecta al número, éste suele marcar el plural morfológicamente mediante el sufijo -reta. Así: sambiai-reta 'niños'; guira-reta 'aves'.

El diminutivo se forma a partir de dos sufijos: -rai y -mi. El primero puede combinarse con nombres, mientras que el segundo puede combinarse con nombres y con verbos: en este último caso, funciona como atenuante de la acción.

y del Madre de Dios (Combès, 2015c).

2 Para la transcripción de palabras en guaraní chaqueño, adopté el alfabeto utilizado en las obras más recientes (Gustafson, 2014; Harwood, 2003 [1995]; Ortiz García y Caurey, 2011; Romero Yaguari, 2006), compuesto por doce vocales, seis orales $(\langle a\rangle,\langle e\rangle,\langle i\rangle,\langle 0\rangle$, $\langle\mathrm{u}\rangle,\langle\dot{\mathrm{i}}\rangle)$ y seis nasales $(\langle\ddot{\mathrm{a}}\rangle,\langle\ddot{\mathrm{e}}\rangle,\langle\ddot{\mathrm{i}}\rangle,\langle\ddot{\mathrm{o}}\rangle,\langle\ddot{\mathrm{l}}\rangle,\langle\ddot{\mathrm{t}}\rangle)$ y dieciocho consonantes simples y compuestas, orales $(\langle\mathrm{ch}\rangle,\langle\mathrm{gu}\rangle,\langle\mathrm{j}\rangle,\langle\mathrm{k}\rangle$, $\langle\mathrm{ku}\rangle,\langle\mathrm{p}\rangle,\langle\mathrm{pu}\rangle,\langle\mathrm{r}\rangle,\langle\mathrm{s}\rangle,\langle\mathrm{t}\rangle,\langle\mathrm{v}\rangle,\langle\mathrm{y}\rangle)$ y nasales $(\langle\mathrm{m}\rangle,\langle\mathrm{mb}\rangle,\langle\mathrm{n}\rangle$, $<\mathrm{nd}>,<\mathrm{ng}>$, $<\tilde{n}>$ ). 
Así, se dice mimba-rai 'animalito'; tëta-mi 'pueblito', o a-karu-mi 'como un poquito'. El aumentativo, por el contrario, se marca ya no mediante el uso de un sufijo sino mediante el uso de lexemas como guasu 'grande'. Por ejemplo: ivitu guasu 'gran viento', o arete guasu 'fiestón', i. e., 'carnaval'.

Existen varios tipos de nominalización. El primero de ellos se da mediante el prefijo mbae-, que se utiliza para formar nombres a partir de bases verbales o nominales (Dietrich, 1986: 180). Por ejemplo, tomemos el caso de la palabra kia 'sucio/a': si se la combina con -mbae- en la forma mbae-kia, se obtiene una palabra que podría traducirse como 'suciedad'. También existe un proceso similar en el que se utiliza el prefijo tembi- (en contexto oral) o temi- (en contexto nasal). Así, del verbo apo 'hacer' junto a este nominalizador obtendremos el nombre tembiapo, que podríamos traducir como 'tarea', 'quehacer', 'trabajo', literalmente, 'lo que se hace'. De acuerdo con Dietrich (1986: 181), estas construcciones se diferencian de aquellas que utilizan mbae- por tener una función sintáctica pasiva. Por último, puede utilizarse el sufijo de función atributiva -vae (Dietrich, 1986: 343) que funciona del siguientemodo: p̈̈ta-vae 'el/la que es colorado/a'.

Es importante, a la vez, tener en cuenta en el proceso de formación de palabras el prefijo poro-, que extiende la acción de un verbo a un objeto humano genérico. Tomemos el caso de mbori 'ayudar', y porombori 'ayudar a todos (acción extendida)'. El reflexivo se marca mediante el prefijo ye- (en contexto nasal ñe-). Por ejemplo, poano 'sanar', y ye-poano 'sanarse', o pisi 'agarrar', y ye-pisi 'agarrarse'. En lo que se refiere al locativo, se marca sumando el sufijo - pe a un topónimo o cualquier nombre que denote la idea de espacio físico. Así, se dice ñemboerenda-pe 'en la escuela', kaa-pe 'en el monte'.

La negación de un lexema se puede marcar mediante el uso de dos sufijos. Por un lado, -mbae que indica ausencia de lo referido, por ejemplo: iya 'dueño', e iya-mbae 'sin dueño'. Por el otro, - $a$, que niega el contenido del lexema, así: ikavi 'bueno', e ikavi-a 'no bueno' (Dietrich, 1986: 179). Existe paralelamente otro tipo de negación típicamente chiriguano -también presente en otras lenguas como el tapiete (Ciccone 2015)- que no se marca mediante un sufijo como las anteriores sino mediante el uso del vocablo mbaeti, que puede expresar la idea de 'no', 'no hay', 'nada', 'inexistente', pero que también puede aplicarse a una acción que se niega: por ejemplo, comparar mbaeti kambi 'no hay leche' con mbaeti a-raja kambi 'no llevo leche'.

Podemos mencionar, además, algunos procedimientos mediante los cuales es posible marcar morfológicamente el tiempo nominal; es decir, la expresión de la referencia temporal ligada con el nombre o la frase nominal (Nordlinger y Sadler, 2004). En el caso del pasado, éste se marca en el nominal con la adhesión del sufijo -kue, que está relacionado con la idea de "ruptura" con un estado original (Dietrich, 2011: 67). Por ejemplo: timbiu 'comida' y timbiu-kue 'lo que fue comida'. Del mismo modo, el futuro nominal suele marcarse con el sufijo -rä: koo 'cerco' y koo-rä 'lo que será cerco'.

La posesión se construye anteponiendo un pronombre personal a lo que es poseído. Las raíces nominales marcadas por una serie de prefijos que pueden funcionar como sujetos, como parte de un atributo o bien de un complemento circunstancial, o también incluso como predicativos (Dietrich, 2009) ${ }^{3}$. De esta forma, se dice che-po 'mi mano', nde-po 'tu mano', i-po 'su mano', ñande-po 'nuestra mano'(inclusivo), ndore-po 'nuestra mano' (exclusivo), pende-po 'su mano', i-po(-reta) 'sus manos'. Al combinarse estos prefijos con algunos nombres cambian la consonante $t$ - inicial propia de su forma independiente por la forma $r$ - en las primeras y en las segundas personas, mientras que en las terceras adoptan la forma $j$-. Esta clase de nombres aparece descripta en los estudios guaraníes como palabras "oscilantes" (Guasch, 1996 [1956]: 65; Krivoshein de Canese y Acosta Alcaraz, 2001: 50; Cerno, 2011: 109). Tomemos para ejemplificarlo la palabra tugui 'sangre': che-r-ugui 'mi sangre', nde-r-ugui 'tu sangre', j-ugui 'su sangre', ñande-r-ugui 'nuestra sangre', ore-r-ugui 'nuestra sangre', pende-r-ugui 'su sangre', j-ugui(-reta) 'su sangre'. También en el caso en el que dos nombres estén conectados por una relación de posesión, el poseedor se manifiesta antes de lo que es poseído por medio de este proceso de "flexión relacional" (Dietrich, 2009: 337).

\section{Los procesos innovación léxica en los registros más tempranos}

Los procesos de innovación léxica no son recientes en el guaraní chaqueño, sino que ya pueden observarse claramente en los primeros registros escritos de esta lengua dejados por misioneros franciscanos a fines del siglo XVIII. Es importante recordar que las poblaciones guaraní hablantes del Chaco occidental se encontraban atravesando por profundos procesos de cambio social desde fines del siglo XVII, como consecuencia de su integración al régimen misional: en un primer momento, a manos de la Compañía de Jesús -la cual inicia, con escaso éxito, la tarea de fundar reducciones en la zona (Langer, 2009)-y, posteriormente, desde mediados del siglo XVIII y hasta mediados del XX, en manos de la Orden Franciscana (Combès, 2015a). Los cambios que atravesó la vida de los hablantes como efecto del proceso de misionalización y posterior incorporación al mercado laboral, así como también por el contacto cada vez mayor con los objetos obtenidos de los criollos, dejaron una huella lexical clara que puede rastrearse desde las antiguas

3 1SG POS che-,2SG POS nde-/ne-, 3SG POS i-/j- o iñ-/iy-, 1PL INCL POS ñande-/ñane-, 1PL EXCL POS ndore-, 2PL POS pende-/pe-, 3PL POS i-/j- o iñ-/y- (Dietrich, 2009). 
fuentes misioneras (Morando, 2018).

Entonces, ya a fines del siglo XVIII podemos observar la emergencia de palabras nuevas en los registros escritos sobre la lengua. Tomemos algunos ejemplos del Diccionario Breve Chiriguanae, publicado por el franciscano español Pedro León de Santiago en 1791: yecha-ka 'espejo', tesa-ïru 'anteojos' -en la grafía original yeechaca, tesairu- (respectivamente, León de Santiago, 1791: 269, 237)4. Analicemos un momento la semántica de estas palabras. Por un lado, ye-cha-ka, está compuesta por el reflexivo ye-, el verbo echa 'ver' y el factitivo que se combina con verbos transitivos - $(u) k a$; lo que podría traducirse como 'lo que hace verse'. En lo que respecta a tesa-ïru, está compuesta por tesa 'ojos' y ïru 'compañero', 'acompañante'; es decir, 'el compañero de los ojos'.

También encontramos en esta obra algunas palabras referidas a las armas de fuego, por ejemplo: mbok $a^{5}$, que aparece como neologismo para 'arcabuz'; mboka guasu, vertida como 'cañón'; o mboka miri, ofrecida como traducción de 'pistola' -en la grafía original: mboca guasu, mboca miri (León de Santiago, 1791: 239). La diferencia entre las armas queda marcada por la adhesión de calificativos a mboka: por un lado, el primero refiere a armas de fuego de gran tamaño como el cañón, en el que a mboka se agrega el aumentativo guasu y, por otro, el segundo refiere a otras armas más pequeñas como la pistola, que aquí aparece como mboka miri, en la que a mboka se añade el diminutivo miri ${ }^{6}$. El etnógrafo sueco Erland Nordenskiöld (1922: 128) propone como hipótesis que mboka sería un préstamo temprano del castellano "boca de fuego" o bien del portugués "boca de fogo".

Doroteo Giannecchini, otro franciscano del Colegio de Propaganda Fide de Tarija que trabajó con las poblaciones indígenas del Chaco occidental, también dejó asentadas en su obra lexicográfica (1916 [1896]) algunas palabras nuevas, como mbae-recha-ka 'catalejo', kore-poti 'dinero' o 'moneda', kore-poti-r'ru iya 'cajero'-en la grafía original: mbaerechaca, corepoti, corepotiriru iya(Giannecchini, 1916 [1896]: 160, 26, 27).

En lo que se refiere a la palabra mbae-recha-ka 'catalejo', está compuesta por mbae 'cosa', el verbo echa 'ver' y el factitivo -(u)ka; o, de ahí que el término pueda glosarse como 'la cosa que hace ver'. Por su parte, kore-poti

\footnotetext{
4 Registradas con anterioridad, por ejemplo, en el Arte y vocabulario de la lengua guaraní (1640) del jesuita Ruiz de Montoya como ñêañgechacába (366) y teça irú (169).

5 Esta palabra aparece ya registrada en Tesoro de la lengua guarani (1639) de Ruiz de Montoya como mbocá 'arcabuz' (215. f.2). Compárese también con las palabras registradas para la lengua tupí en el siglo XVII en el Vocabulário na Língua Brasílica escrito en 1622 presumiblemente por Pero de Castilho (1952-1953 [1622]): tatâigba 'fuzil de ferir fogo', yapígya 'fozil de cadea' (143).

6 Este diminutivo, muy común en el guaraní paraguayo, aparece registrado para el guaraní chaqueño únicamente en esta obra. En las obras escritas posteriormente para esta lengua aparece más bien la forma -mi, que es la utilizada por los hablantes en la actualidad.
}

'dinero' deriva por composición de kuare 'hoyo', 'agujero' y, posiblemente, de tepoti 'excremento', 'evacuación', 'floración', con lo que podríamos glosar la palabra como 'evacuaciones de un hoyo'. La acepción de esta palabra en tanto 'dinero' o 'moneda' aparece registrada por primera vez en la obra seminal de Giannecchini (1916 [1896]: 26), y su uso se mantiene vigente entre los hablantes del guaraní chaqueño hasta el día de hoy, como en el caso específico de kore-poti-r'ru iya 'cajero', que puede analizarse en kore-poti 'metal' o 'dinero', r'ru 'contenedor' e iya 'dueño', y entonces glosarse como 'dueño del contenedor de dinero'7.

En esta obra pueden encontrarse además otras palabras relacionadas con máquinas, como mbae-yopi-ja 'prensa', kuaras'-r-aanga 'reloj' o takua-nëe yoso-ja 'trapiche' -en la grafía original: mbaeyopiha, cuarassiraanga, tacunée iyosoha-(Giannecchini, 1916 [1896]: 142, 154, 177). En el caso de mbae-yopi-ja, palabra para designar a la 'prensa', está compuesta por mbae 'cosa', yopi 'comprimir', 'aplastar' y el sufijo agente -ja, que indica el ejecutor de una acción; por lo que podríamos traducirla como 'la cosa que comprime o aplasta'. Por su parte, la palabra kuaras'-r-aanga está compuesta por kuaras' 'Sol' y taanga 'medida', 'imagen' (cuya forma flexionada con marcador posesivo es raanga), con lo que podríamos traducirla como 'la medida del Sol'. En relación con la palabra takua-nëe yoso-ja 'trapiche', podemos observar que está formado, por un lado, por takua-nëe 'caña dulce' y, por el otro, por yoso-ja, donde yoso significa 'moler' y -ja es el sufijo agente; de donde resulta la glosa 'lo que muele la caña dulce'. Hasta ahora, todas las palabras revisadas parecen ser palabras nuevas creadas conscientemente en base a recursos morfosintácticos propios de esta lengua.

Asimismo, en otros materiales misionales del mismo período pueden observarse una gran cantidad de palabras relacionadas con la religiosidad católica, a consecuencia del avance incontenible del proyecto evangelizador. Ya que esto ha sido tratado extensamente para el caso chiriguano (Villar, 2008; Morando, 2017) o también para el caso del guaraní oriental (Otazú, 2006; Boidin, 2016; Cerno, 2016), aquí señalaré solamente algunos ejemplos que pueden ser útiles para ilustrar la cuestión. Tomaré para ello las palabras Tumpa y Aña, con las cuales se encontró en la lengua indígena una equivalencia para las ideas cristianas de 'Dios' y 'diablo', respectivamente. Se trata de claros ejemplos de extensión semántica, ya que ambos términos existían originalmente en la lengua. En cuanto a Tumpa, aparece ya registrada para el guaraní occidental en el siglo XVII por el jesuita Ruiz de Montoya (1639: 402, f. 2), que la analiza como tû 'admiración' y

\footnotetext{
7 Contrástese con el caso del guaraní paraguayo, en que la palabra kuarepoti significa 'metal', pero para referirse al 'dinero' se utilizan más bien pirapire (literalmente 'escama de pescado') o viru (proveniente del castellano viruta, que asimismo se utiliza informalmente en algunos países hispano hablantes para designar al 'dinero').
} 
pã 'pregunta' -en la grafía original, Tu'pa'8. Mucho más tarde, ya en descripciones sobre el guaraní chaqueño, por ejemplo, Doroteo Giannecchini (1869: f.1) relata lo siguiente con respecto al sentido indígena original de la palabra: "significa un quid grande ideal; una cosa real que excita admiración; una cosa inexplicable, ininteligible; una cosa extraña, maravillosa; una cosa que hiere la fantasía y los sentidos". Lo mismo sucede con la palabra Aña, registrado también por Ruiz de Montoya (1639: 42, f. 2) para el guaraní oriental como âng 'alma' y yñã 'correr' -en la grafía original, añãng. Al igual que lo que ocurre con el caso de Tumpa, Giannecchini (1869: f. 2) logra ilustrar claramente los múltiples sentidos que los hablantes otorgaban a aquella palabra: "todo lo que tiene aspecto de feo y de mal [...]; los sueños, las pesadillas". Al contrario de las palabras previamente revisadas, Tumpa y Aña no sólo evidencian un proceso de extensión semántica, sino que también representan un claro ejemplo de aquello que Boidin $(2016,2017)$ denomina middle ground; "un espacio de comunicación y acción común" entre misioneros e indígenas ${ }^{9}$.

El proceso de registro de nuevas palabras por los misioneros franciscanos puede seguirse hasta las primeras décadas del siglo $X X$, cuando por una serie de circunstancias -secularización de las misiones en Bolivia, comienzo de la Guerra del Chaco, migraciones masivas de indígenas desde Bolivia hacia la Argentina para trabajar en la industria agrícola y azucarera (Bossert, Combès y Villar, 2008; Langer, 2008; Métraux, 1930a; Riester, 2008)- el régimen misional queda severamente afectado y comienza a declinar la producción escrita de los misioneros, progresivamente sucedida por los aportes de etnógrafos y lingüistas (Nordenskiöld, 2002 [1912]: 147; Schmidt, 1938).

Podría remitirme, por ejemplo, a las descripciones que el etnógrafo suizo Alfred Métraux (1930b: 380-382) hace sobre el uso de la balanza entre indígenas guaraní hablantes chaqueños, a la que según sus informaciones se refieren mediante el préstamo del castellano mbalansa -o en la grafía original mbalanza-, en el que puede observarse la adaptación del fonema castellano /b/ al fonema guaraní chaqueño $/ \mathrm{mb}$. O bien a las extensas compilaciones de Erland Nordenskiöld de nuevas palabras surgidas entre distintos grupos indígenas sudamericanos como consecuencia del contacto con el blanco. Para el caso de guaraní chaqueño, el sueco incluye también el castellanismo kavayu 'caballo' -en la grafía original, cavayu (Nordenskiöld, 1922: 58).

Dentro del conjunto de trabajos lingüísticos escritos

8 Esta palabra, incluso, puede encontrarse en materiales tupí anteriores -por ejemplo, Thévet (1586: 253); De Oré (1607: 415-416)-, de los cuales posiblemente haya sido tomada por Montoya y luego por los misioneros franciscanos del Chaco occidental.

9 Compárese, por ejemplo, con el análisis de terminología cristiana y su incorporación a la lengua estudiada detalladamente para el caso wichí por Montani (2017). durante este período, también podría citarse el del anglicano británico Leslie Frank Harwood, que entre 1928 y 1935 trabajó en la región boliviana del Isoso como miembro de la South American Missionary Society (Combès, 2015b; Córdoba, 2019) y confeccionó un diccionario, publicado recién póstumamente, en 1995: Ñaneñee Riru (Harwood, 1995; Morando, 2018). Menciono este trabajo en particular porque, si bien en la versión original no existían referencias a nuevas palabras, estas sí aparecen en la última versión del mismo editada en el año 2003 (Harwood, 2003 [1995]) por el Comité Hable Guaraní, una organización indígena surgida en 1983 cuyo objetivo era la divulgación de la lengua.

\section{Los procesos de innovación léxica en las últimas cuatro décadas}

Los procesos de innovación léxica se acentúan a partir de la década de 1980, como efecto de una serie de cambios que suceden en la organización indígena en Bolivia luego de que en 1987 se decidiera reconocer al guaraní, el aymara y el quechua como lenguas oficiales de la nación junto con el castellano. Fruto de ello, en 1987 se crea la Asamblea del Pueblo Guaraní (APG), órgano máximo de representación del pueblo guaraní en este país y, asimismo, otras organizaciones como el ya mencionado Comité Hable Guaraní (Hirsch, 2003; Admiraal, 2006). Paulatinamente, el guaraní chaqueño comienza a cobrar una mayor presencia en distintos ámbitos de la vida social y política regional -escolar, jurídico, académico-, lo que dio lugar al surgimiento de nuevas palabras por medio de distintos mecanismos. Estas palabras han quedado plasmadas en materiales escritos que circulan entre la población guaraníhablante del sureste boliviano y el Noroeste Argentino ${ }^{10}$.

\section{El dominio jurídico, social y cotidiano}

Gran parte de los cambios que comienzan a sucederse a partir de la década de 1980 en la organización de la estructura del guaraní chaqueño están vinculados con la creciente participación indígena en el ámbito jurídico. Como explica Scionti (2016), a partir de este momento el sistema de autoridad y justicia guaraní tradicional -en el que cualquier decisión de este tipo recae sobre la yemboati 'asamblea' (Hirsch y Alberico, 1996)- se articula de forma definitiva con los ordenamientos oficiales de los Estados Nacionales en los que se encuentran emplazada esta comunidad de guaraní hablantes. En Bolivia, incluso, la propia posibilidad de ejercicio y de administración de la justicia indígena está contemplada a partir del año 2009 en la Constitución Nacional (Constitución Política del Estado Plurinacional de Bolivia: Parte II, Título III, Capítulo IV).

\footnotetext{
10 Compárese con lo sucedido en el caso paraguayo. En 1967 se reconoce al guaraní como lengua nacional pero no fue sino hasta 1992 que fue reconocida como oficial. Esto propulsó su inclusión en ámbitos en los que, hasta ese momento, no había podido penetrar, como el contexto educativo, académico, administrativo y/o político (Boidin,1999; Choi, 2003).
} 
Por ello reservo este primer apartado para registrar una serie de palabras nuevas que han surgido en la esfera del derecho y lo jurídico. Tomaré, por ejemplo, el caso de yeyora, palabra por medio de la cual se ha dado traducción en la lengua indígena al concepto occidental de 'libertad' (Harwood, 2003 [1995]: 410). Esta palabra está conformada de la siguiente manera: la raíz proviene del verbo yora, que tiene una gran variedad de significados, entre ellos 'desatar', 'largar' o 'abrir', al que luego se ha adicionado el reflexivo ye-; por lo que una glosa literal de yee-yora podría ser 'desatarse', 'largarse', 'abrirse'. Otra de las palabras que resulta interesante rescatar es ye-mbosimbi, que traduce el concepto castellano de 'disciplina' (Harwood, 2003 [1995]: 371). Al igual que ye-yora, la misma está compuesta por el reflexivo ye-, y el verbo mbosimbi, literalmente 'rectificar', con lo que podríamos traducirla aproximadamente como 'lo que rectifica'. Podría incluirse, también, el neologismo teko-mboete-ka, empleado para referir a los 'derechos', y compuesto por teko 'costumbre' o 'modo de vida', mboete 'respetar' y el factitivo -(u)ka, lo que podría traducirse literalmente como 'lo que hace respetar la costumbre o el modo de vida'.

Entrando ya en lo propiamente jurídico, puede mencionarse asimismo la palabra poro-kuai, utilizado como traducción del concepto legal occidental de 'ley', 'orden' o 'norma' (Ayma et al., 2004: 50). En la base de la palabra se encuentra el verbo yokuai 'mandar' u 'ordenar', modificado por el prefijo de objeto humano genérico incorporado poro-; es decir, 'aquello que manda u ordena a la gente'. Estrechamente relacionado con poro-kuai está poro-kuai guasu, neologismo en el que a poro-kuai se añade el aumentativo guasu y que se utiliza para referir a una 'constitución política'.

Dentro de este repertorio podemos citar también algunos casos de extensión semántica. Por ejemplo, lo que está 'fuera de la ley' o, en otras palabras, lo 'ilegal', se designa a partir de la expresión ikavi-a mbae, que literalmente significa 'cosa que no está bien', y de ahí, por ejemplo, jae ikavi-a mbae u-yapo 'él/ella hizo algo malo' o kuae kuña j-eko ikavi-a mbae, 'esa mujer no lleva una buena vida'. Lo que sucede aquí es una ampliación semántica del sentido original de la expresión ikavi-a mbae 'cosa que no está bien', que se ha adaptado al contexto jurídico. La 'claridad' o 'transparencia', en el sentido institucional, también ha sido traducida como tembipe (Ayma et al., 2014:15). Esta palabra ya existía en la lengua, con el sentido original de 'claridad lumínica'; se trata, pues, de un caso de extensión semántica en el que una palabra ya existente en la lengua nativa adquiere un nuevo significado para adecuarse al de otra proveniente del castellano.

Asimismo, podrían incluirse dentro de la categoría de las extensiones semánticas otras palabras relacionadas con símbolos ciudadanos. Por ejemplo, la idea de 'escudo' o 'blasón', en tanto símbolo de un estado o una organización, ha sido traducida por medio de una palabra ya existente: yeopiaka (Ayma et al., 2004: 33), que en su sentido más literal refiere a un 'escudo' como elemento de protección. Otro símbolo de la sociedad civil que también encuentra traducción es el 'himno': pirae guasu; a la palabra pirae 'canto' se le suma el aumentativo guasu para dar la idea de un 'canto grande'.

Además, mencionaré algunas palabras relacionadas con la vida cotidiana que también nos permiten repensar los procesos de innovación léxica. Como veremos, aquí se nota una prevalencia de préstamos del castellano. Esto resulta claro, por ejemplo, si tomamos los nombres de algunos alimentos incorporados a la dieta indígena como consecuencia del contacto con el frente colonizador y que se encuentran altamente estabilizados por su uso corriente. Así, tenemos: suká (préstamo del castellano "azúcar"), aró (préstamo del castellano "arroz"), kesiyu (préstamo del castellano "quesillo"), nará (préstamo del castellano "naranja"), neche (préstamo del castellano "leche"). En los casos de aró, nará o suká, además de la adaptación de la grafía al alfabeto guaraní chaqueño, se observa principalmente una reducción fónica de las palabras castellanas, ya sea hacia el final (apócope) o hacia el comienzo (aféresis). Tomaré, por último, el caso de algunos préstamos que se originan en verbos castellanos y que aparecen combinados con afijos de la lengua indígena, algo que ha sido observado asimismo para el caso del guaraní paraguayo (Zajícová, 2009), aunque en el guaraní chaqueño los verbos no sólo aparecen combinados con marcas de persona provenientes de la lengua indígena sino también con el factitivo mbo- (en contexto nasal mo-). Así, por ejemplo: che a-mbo-le 'yo leo', che a-mbo-escribe 'yo escribo' -que alterna con la variante indígena kuatia. Algo similar sucede con el verbo 'poder' del castellano, que ha pasado al guaraní chaqueño como puere; así, por ejemplo, se dice che puere 'yo puedo'.

\section{El dominio escolar y académico}

Últimamente, en la esfera del léxico pedagógico y académico ha surgido también una gran cantidad de nuevas palabras, sobre todo como consecuencia de proyectos educativos interculturales y bilingües en la Argentina y en Bolivia, y de la subsiguiente necesidad de adaptar las currículas escolares a la lengua indígena. Resumiré algunos nombres que, en líneas generales, se ligan con lo educativo en la lengua indígena de acuerdo con lo que he podido relevar durante mi trabajo de campo entre los chanés del Noroeste Argentino y los guaraníes del sudeste boliviano, como también en algunos documentos publicados (para el caso boliviano: Asamblea del Pueblo Guaraní [APG], 2014; para el caso argentino: Consejo Continental de la Nación Guaraní [CCNAGUA] et al., 2018). Así, por ejemplo, tomando como base la palabra arakua 'saber' o 'conocimiento', se han creado diferentes palabras que refieren a las distintas áreas temáticas de la currícula escolar. Por ejemplo, 
arakua-ivipo-reta como traducción para el área de estudio denominada 'ciencias de la tierra', donde arakua se traduce como 'conocimiento', ivipo que refiere a 'faz de la tierra y lo contenido en ella' (Giannecchini, 1916 [1896]: 80) y -reta es la marca de plural; es decir, entonces, 'los conocimientos de la tierra y lo contenido en ella'.

Otra área dentro de este conjunto léxico son las arakuatekove-regua o 'ciencias de la vida'. Esta palabra está compuesta por arakua 'conocimiento', tekove 'vida' y regua (cuya forma no flexionada con pronombre personal es pegua), sufijo que de acuerdo con Dietrich (1986: 139) funcionaría como un complemento circunstancial de causa; de ahí que una buena traducción literal del compuesto pueda ser 'acerca del conocimiento de la vida'. Por último, también he relevado una palabra que refiere a las 'ciencias sociales' muy similar al anterior: arakua-teko-reta. Esta palabra está compuesta por arakua 'conocimiento', teko 'costumbre', 'modo de vida' y el plural -reta; con lo cual podría glosarse, aproximadamente, como 'los conocimientos de las costumbres'.

En este proceso de traducción de la currícula oficial a la lengua indígena también se han definido áreas como ñemboe kia che o 'educación para la identidad', donde ñemboe significa 'aprender', kia es un pronombre que significa 'quién' y che es el pronombre de primera persona del singular. Esto podría traducirse, entonces, como 'aprender quién soy yo'. Otra área de estudio traducida a la currícula escolar es 'educación para la salud' o ñemboe teko-kavi-väe-rä. Este nombre está compuesto por ñemboe 'aprender' y teko-kavi-väera: teko-kavi 'buenas costumbres', 'buen modo de vivir' y el sufijo nominalizador -väera, que incluye la marca de futuro nominal -rä (Dietrich, 2009: 358); esto lo podríamos traducir aproximadamente como 'aprendizaje del buen modo de vivir'.

También existe una nueva palabra curricular para 'educación para la economía': ñemboe kavi mbaravikire, nombre compuesto por el verbo ñemboe 'aprender', el adjetivo kavi 'bueno', mbararaviki 'trabajo' más el sufijo -re 'por', 'sobre'; literalmente, 'aprender sobre el buen trabajo'. Existe, por otro lado, una nueva palabra para referirse al área de la matemática: papaka-regua. Está compuesta por papa 'contar números', el factitivo -(u)ka sumado a regua (cuya forma no flexionada con pronombre personal es pegua) que opera como complemento circunstancial de causa (Dietrich, 1986: 139); o, en otras palabras, 'acerca del contar o de las cuentas' (Aruchari et al., 1996).

Puede observarse además un vocablo para designar el área curricular 'literatura': kuatia-pöra. Esta palabra está compuesta por el verbo kuatia 'escribir' (pero también 'libro') y la contracción del adjetivo maëpora 'bonito'; es decir, 'escribir bonito'. Alternativamente podría mencionar una nueva palabra para nombrar el área de 'lengua y cultura': ñee jare ñande-r-eko (cuya forma no flexionada con pronombres posesivos es teko), literalmente, 'nuestra lengua y costumbres o modo de vivir'.

Por último, trataré algunas nuevas palabras que tienen que ver con espacios de aprendizaje. En guaraní chaqueño existen dos palabras para nombrar la 'escuela': ñemboe-a y ñemboe-renda. La primera está compuesta por ñemboe 'aprender' y la marca de lugar - $a$, lo que podemos traducir aproximadamente como 'lugar donde se aprende', mientras que la segunda está compuesta por ñemboe sumado a tenda 'lugar' o 'sitio' flexionada en función atributiva, lo que podemos traducir del mismo modo. Nemboe- $a$ no es una palabra nueva ya que aparece en los registros de Léon de Santiago (1791: 296) y Giannecchini (1916 [1896]: 70). Ñemboe-renda aparece registrada posteriormente en la última edición del diccionario de Harwood (2003 [1995]: 382). Partiendo de aquella base, se han creado otras palabras durante las últimas décadas como ñemboe-renda guasu 'universidad', donde a la palabra ñemboe-renda se le adiciona el aumentativo guasu. A su vez, al igual que el 'director' de una escuela lleva el nombre de ñemboe-renda tuvicha y el 'rector' de una universidad, consecuentemente, llevará el de ñemboe-renda guasu tuvicha. En este caso, a ñemboerenda y a ñemboe-renda guasu se añade el nombre tuvicha 'cacique' (de modo más general 'autoridad'); es decir, entonces, 'la autoridad de la escuela' y 'la autoridad de la universidad' respectivamente. Podría incluirse aquí también la palabra poromboevae, neologismo para 'maestro/a' o 'profesor/a' y que está formada por el prefijo de objeto humano genérico incorporado poro- sumado a mboe 'enseñar' y el sufijo de función atributiva -vae, lo que podría traducirse aproximadamente como 'el/la que enseña'. Lo que advertimos en primera instancia en este dominio léxico es una predominancia casi total de palabras nuevas que han sido creadas a partir de elementos provenientes de la propia lengua en detrimento de los préstamos del castellano o de la adaptación de palabras ya existentes en la lengua.

El dominio de los sistemas de medición y sus unidades Dentro del repertorio de nuevas palabras surgidas a partir de las últimas décadas del siglo $X X$, pueden relevarse también -aunque todavía en menor medidaalgunos neologismos que refieren a unidades métricas. Por ejemplo, para el caso de las unidades de longitud, podemos citar ye-jaä-ta-popa, traducción que se le ha encontrado en esta lengua al concepto de 'centímetro'. Esta palabra está compuesta por el reflexivo ye-, el verbo jaä 'medir', la marca de futuro -ta más la contracción del numeral cien (pañandepopa), lo que podríamos traducir como 'lo que se mide la cantidad cien'. Lo mismo ocurre con la palabra que se ha utilizado como equivalente del concepto de 'metro', ye-jäa-ta. La única diferencia aquí es que solamente está compuesto por el reflexivo ye- más el verbo jaä; es decir, 'lo que se mide'. Por último, el 
concepto de 'kilómetro' ha surgido como jeta-yejäa-ta. La construcción es similar a las anteriores, a excepción del hecho de que la palabra comienza con jeta 'muchos' (Ayma et al., 2004: 14, 55).

Por otro lado, en lo que se refiere a las unidades de peso, se observa un neologismo para el concepto de 'kilo', jetakure. Esta palabra está compuesta por jeta 'mucho/s' y kure 'material a granel', lo que podría traducirse entonces como 'mucho de material a granel'. Existe también una palabra nueva para la unidad de capacidad 'litro': ti-jäa formada por tigue 'líquido' y jaä 'medir': 'la medida del líquido' (Aruchari et al., 1998: 23).

Por último, acerca de las unidades de tiempo, podemos decir que existen en esta lengua algunas palabras tradicionalmente indígenas para nombrar determinadas unidades temporales correspondientes con lo meses del año que no parecerían implicar necesariamente una innovación ${ }^{11}$. Podemos relevar otra unidad que se corresponde con un 'año': ara-sa, palabra compuesta por ara 'tiempo' más la contracción del verbo asa 'pasar'. Tomo para ejemplificar esto un fragmento de un relato autobiográfico que registré del cacique chané Taparindu de Tuyunti, en el que relata su vida personal: chiu arasa a-reko yae, täta a-vata, chiu ara-sa a-iko yae, che ruvicha katu yae a-a escuela-pe, es decir, 'ya tenía siete años, caminaba fuerte. Siete años tenía en ese momento. Cuando era más grande ya fui a la escuela' (Morando, 2020: 342). Ahora bien, no puedo asegurar en este caso si se trata de una nueva palabra -el primer registro en el que pude identificarla es Harwood (1996 [1995]: 130)-, pero sí que se encuentra incorporada al léxico cotidiano y puede escucharse comúnmente, por ejemplo, en relatos históricos y/o autobiográficos.

Existen, además, algunas unidades temporales menores, ya sí designadas mediante nuevas palabras creadas a lo largo de los últimos años en base a recursos propios de la lengua, como ara-jai 'hora' (Harwood, [1995]: 398),

11 Si bien existen entre los distintos dialectos de esta lengua existen algunas variantes en la adjudicación de estos nombres, tomaré a continuación solamente el caso de la variante dialectal chané a fines prácticos : yasi-pi 'enero', ore-ti 'febrero', yaviye 'marzo', ara-ivi 'abril', ara-ivo 'mayo', ara-roi 'junio', ara-tini 'julio', ara-ivitu 'agosto', arapoti 'septiembre', ara-kuvo 'octubre', ara-ama 'noviembre', ara-eti 'diciembre'. Algunos de estos nombres están compuestos mediante combinación con yasi 'luna', mientras que otros se forman a partir del concepto genérico de ara 'tiempo', sumado a alguna característica climática que se corresponde con cada momento específico del ciclo anual sobre las cuales no entraré en detalle aquí. Sólo por tomar dos ejemplos: yasí-pi 'enero', esta palabra está compuesta por yasi 'Luna' e ipi 'comienzo' u 'origen'; dada la relación entre el ciclo lunar y el mes, podríamos traducirlo como 'mes inicial'. En el caso de ara-ivi 'abril', esta palabra está compuesta por ara 'tiempo' y jaivi 'llovizna'; es decir, 'tiempo de llovizna'. Compárese, por ejemplo, con lo ocurrido en el guaraní paraguayo. En esta lengua el proceso de formación de los nombres de los meses se da exclusivamente mediante la palabra jasy 'Luna'/ 'mes', combinado con números cardinales: jasyteĩ 'enero' (jasy + peteĩ 'uno'= 'mes uno'), jasykõi 'febrero' (jasy + mokõi 'dos'= 'mes dos'), y así sucesivamente (Ateneo de Lengua y Cultura Guaraní, 2006: 68). compuesta por ara y jai, 'fragmento' o 'pedazo'; es decir, 'fragmento' o 'pedazo de tiempo'. El 'minuto', por su parte, lleva el nombre de ara-jai-mi (Ayma et al., 2004: 56), en que a ara-jai se agrega el diminutivo -mi, es decir, 'un pequeño fragmento o pedazo de tiempo'. Al contrario de lo que sucede con el caso de ara-sa, estas dos palabras ya no tienen una presencia tan común en el habla cotidiana.

\section{El dominio mecánico}

Por último, reservaré un espacio particular para las palabras relacionadas con el dominio mecánico. Si bien no existe una palabra específica para designar la idea misma de 'máquina', ésta suele nombrarse mediante la palabra tembi-poru, categoría que también puede utilizarse para designar cualquier tipo de 'instrumento', 'artefacto' o 'herramienta'. En un sentido estricto, esta palabra está compuesta por el prefijo nominalizador tembi- más el verbo poru 'usar', con lo que podría entenderse de manera literal como 'lo que se usa'. Por otra parte, el conjunto de conocimientos en base a los cuales funcionan las máquinas o 'tecnología' también se encuentra lexicalizado bajo la palabra mbae-pokiapo (Ayma et al., 2004: 85). Esta palabra está formada por mbae 'cosa', poki 'habilidad' y finalmente el verbo apo 'crear', con lo cual podríamos traducirla de manera aproximada como 'cosa que crea habilidad'.

En lo que se refiere a nombres de máquinas específicas, puede citarse también el 'torno', que en esta lengua recibe el nombre de yere-ka, palabra que proviene del verbo yere 'girar' sumado al factitivo -(u)ka, con lo cual podría traducirse como 'lo que hace girar'. Tómese como ejemplo la 'bomba', cuyo nombre en guaraní chiriguano es mbo-yere moata. Al igual que yere-ka, mbo-yere moata proviene del verbo yere 'girar' más el factitivo -mbo- y moata 'atraer', con lo cual equivaldría a 'hacer girar y atraer'. Otro tipo de máquina que podría incluirse es, por ejemplo, el 'automóvil', que lleva el nombre de täkarara estrechamente ligado con el verbo täkarara o tökororo 'rodar'.

Pueden mencionarse, por fin, algunos otros nombres de máquinas cuya incorporación ha sido quizá más reciente, como por ejemplo el 'teléfono'. A este artefacto se lo encuentra traducido como ñee-rape. Esta palabra está compuesta por ñee, que puede glosarse como 'lenguaje', 'idioma', 'voz' o 'habla' y luego por tape 'camino'. Esto podría traducirse consecuentemente como 'el camino del habla'. Otro artefacto para el que existe un neologismo en esta lengua es la 'radio' o ñee-rendu-ka (Veliz, 1999: 145). Esta palabra está compuesta por ñee 'lenguaje', 'voz', 'habla', el verbo endu 'escuchar' más el factitivo -(u)ka; es decir, entonces, 'aquello que hace escuchar la voz, el habla'. Por último, también podría incluirse en este conjunto a kuatia-siri-ka (Ayma et al., 2004: 17), una nueva palabra para nombrar la 'computadora'. Esta palabra está compuesta por el verbo kuatia 'escribir', la 
contracción del adjetivo siriki 'ágil', 'veloz' sumado al factitivo -(u)ka; 'aquello que hace escribir velozmente'.

Hasta aquí, todos los nombres de máquinas mencionados se nos presentan como nuevas palabras, sin embargo, podemos incluir aquí también el caso de vehículos como el camión que, en cambio, toman su nombre del castellanismo kamiö. Aquí, por un lado, se produce la pérdida de la consonante final $/ \mathrm{n} /$ y la consecuente nasalización de la vocal /o/ que queda en última posición y se mantiene el acento prosódico oxítono de la palabra castellana. Por otro lado, desde el punto de vista gráfico, además de marcarse la nasalidad de la última vocal $<$ ö $>$, se produce un cambio de $<\mathrm{c}>\mathrm{a}<\mathrm{k}>$ de acuerdo con el alfabeto del guaraní chaqueño más actual.

\section{Palabras finales}

Los procesos de innovación léxica se manifiestan en el guaraní chaqueño de tres formas diferentes. En primer lugar, por la creación consciente de nuevas palabras basadas en recursos morfosintácticos de la propia lengua como ñee-rendu-ka 'radio', täkarara 'automóvil', porokuai 'ley', papa-ka-regua 'matemática' o teko-mboete$k a$ 'derechos', que surgen sobre todo por composición sobre la base de otras palabras. En segundo lugar, por la incorporación a la lengua de préstamos provenientes del castellano y que han sido adaptados en su fonética, tal como sucede con los casos de suká, aró, kamiö. Por último, la innovación puede ocurrir mediante la extensión en los sentidos de palabras ya existentes; tal es el caso de tembipe 'claridad' en el sentido institucional, tembiporu 'máquina' o yeopiaka 'escudo' en tanto símbolo, ikavi-a mbae algo 'ilegal' o 'fuera de la ley', Tumpa 'Dios cristiano' aunque originalmente 'lo maravilloso o sorprendente', aña 'diablo' aunque originalmente 'espectro' o 'pesadilla', o ara 'paraíso', originalmente 'cielo' y 'tiempo'.

Hemos visto que estos procesos pueden observarse en esta lengua ya desde los primeros registros que datan de fines del siglo XVIII. Pero, sobre todo a partir de la década de 1980, los datos marcan un largo y complejo proceso de adaptación de la lengua indígena a distintas coyunturas sociales e históricas. La mayor prevalencia de las primeras dos operaciones -la aparición de nuevas palabras por composición nominal y extensión de sentidos semánticos de palabras ya existentes-, podría llevar a pensar en aquello que Zimmermann (2006) Ilama "purismo proactivo"12 de ciertos grupos guaraní hablantes (maestros bilingües, representantes de comunidades, indígenas vinculados a agencias estatales u organizaciones indigenistas, etc.). Sin embargo, lo cierto es que, si bien existe una gran cantidad de palabras nuevas creadas constantemente, muchas de ellas no se encuentran del

12 Es decir, la creación consciente y planificada de nuevas palabras, por medio de elementos provenientes de la misma lengua evitando préstamos de otras (Zimmerman, 2006: 508). todo incorporadas al habla cotidiana y su uso queda restringido a la esfera escolar, académica, jurídica o técnica, ligado a un uso tal vez más "culto", "puro" o "correcto" del guaraní chaqueño o chiriguano en un claro esfuerzo de los propios hablantes por "formalizar" o "institucionalizar" su lengua. Ciertamente esto traduce la determinación de posicionamientos étnicos que tienen por objeto la revitalización y revalorización de la lengua nativa. Por ello, lo que es importante rescatar en el caso analizado es el hecho de que los múltiples procesos de innovación léxica se vinculan con un actual proceso de revitalización lingüística y reivindicación identitaria que, desde hace tres décadas, comienza a reforzar cada vez más la presencia cultural guaraní en el Chaco occidental.

Un tema como el abordado aquí no se agota en el mero análisis de un catálogo de palabras, ni tampoco en el desmenuzamiento y disección de las tramas semánticas que las conforman, sino que es imprescindible, además, no desatender el hecho de que todos estos procesos están ligados a cambios coyunturales por los que los hablantes guaraníes chaqueños han atravesado a lo largo de determinados momentos históricos. Una visión más amplia sobre los contextos etnohistóricos en los que se producen estos cambios en la lengua también es necesaria para comprender más acabadamente los diversos modos en los que los indígenas significan su "modernidad" como respuesta al contacto intercultural (Paula Camargo, 2020). Por lo tanto, este tipo de problemáticas requiere un abordaje a partir de una mirada auténticamente interdisciplinaria que permita consolidar una amalgama razonable entre sus matices lingüísticos, antropológicos e históricos.

Martínez, 10 de noviembre de 2020

\section{Agradecimientos}

Agradezco a los doctores Rodrigo Montani, Diego Villar y Cristina Messineo por sus valiosos comentarios y aportes a las versiones preliminares de este artículo, así como también por la exhaustiva revisión de los datos aquí consignados.

\section{Referencias bibliográficas}

Admiraal, F. (2006). Taal en Socialisatie Spaans en Guaraní in Isoso, Bolivia. Tesis de Doctorado, Universiteit van Amsterdam, Amsterdam.

Asamblea del Pueblo Guaraní. (2014). Currículo Regionalizado Guaraní. Documento de Trabajo.

Aruchari, R., H. Urday y E. Gil. (1996). Papakaregua: Ñeekuakua II. Ñemboe Yipi. La Paz: Ministerio de Desarrollo Humano.

Aruchari, R., I. Mesa, H. Urday, S. Camacho, E. Gil y C. 
Lampo. (1998). Papakaregua: Ñeekuakua III. Ñemboe Yipi. La Paz: Ministerio de Desarrollo Humano.

Ateneo de Lengua y Cultura Guaraní. (2006). Guarani mbo'ekuaahára mbo'esyry III. Asunción: ZADA.

Ayma, S., J. Barrientes y G. Márquez. (2004). Arusimiñee. La Paz: Ministerio de Educación de Bolivia.

Boidin, C. (1999). La política de educación bilingüe guaraní-español en el Paraguay de los años 1990. Revista Paraguaya de Sociología, 105, 147-158.

Boidin, C. (2016). Pensar la modernidad/colonialidad en guaraní (XVI-XVIII). Cuadernos de Antropología Social, $44,7-25$.

Boidin, C. (2017). Mots guarani du pouvoir, pouvoir des mots guarani. Essai d'anthropologie historique et linguistique (XIX-XVI et XVI-XIX). Tesis de habilitación, Université Sorbonne Nouvelle, París.

Bossert, F., I. Combès y D. Villar. (2008). La Guerra del Chaco entre los chané e isoseños del Chaco occidental. En N. Richard (Comp.), Mala guerra. Los indígenas en la guerra del Chaco (1932-1935) (pp. 203-234). París/ Asunción: ServiLibro-Museo del Barro/CoLibris.

Brown, C. H. y Witkowski, S.R. (1983). Polysemy, Lexical Change and Cultural Importance, Man, 18 (1), 72-89.

Casado Velarde, M. (2015). La innovación léxica en el español actual. Madrid: Síntesis.

Castilho, P. de. (1952-1953 [1622]). Vocabulário na Língua Brasílica (edición de Carlos Drumond). San Pablo: Carlos Drumond.

Consejo Continental de la Nación Guaraní, Asamblea del Pueblo Guaraní y Consejo Educativo de Pueblos Indígenas. (2018). Currículum Ñande Reko para educación primaria guaraní, chané y tapiete. Salta: Ministerio de Educación de Salta.

Cerno, L. (2011). Descripción fonológica y morfosintáctica de una variedad de la lengua guaraní hablada en la provincia de Corrientes (Argentina). Tesis de Doctorado, Universidad Nacional de Rosario, Rosario.

Cerno, L. (2016). La traducción guaraní de la Conquista Espiritual. Un caso de pragmática transcultural. En M. L. Salinas (Ed.), Actas de las XVI Jornadas Internacionales sobre las misiones jesuíticas (pp. 157-167). Resistencia: IIGHI.

Choi, J. K. (2003). Language Attitudes and the Future of Bilingualism: The Case of Paraguay. International Journal of Bilingual Education and Bilingualism, 6 (2), 81-94.
Ciccone, F. (2015). Contacto, desplazamiento y cambio lingüístico en tapiete (tupí-guaraní). Tesis de Doctorado, Universidad de Buenos Aires, Buenos Aires.

Combès, I. (2005). Etno-historias del Isoso. Chané y chiriguanos en el Chaco boliviano (siglos XVI-XX). La Paz: IFEA.

Combès, I. (2015a). Historia franciscana y etnografía chiriguana. Boletín Americanista, 70, 57-72.

Combès, I. (2015b). Una experiencia anglicana en el Chaco boliviano (1926-1935). Boletín Americanista, 70, 135-158.

Combès, I. (2015c). Itatines y Guarayos (Oriente boliviano, siglos XVI-XVIII).Anthropos, 110, 361-382.

Combès, I. y T. Saignes. (1995). Chiri-guana: nacimiento de una identidad mestiza. En J. Riester (Eds.), Chiriguano: Pueblos indígenas de las tierras bajas de Bolivia (pp. 25221). Santa Cruz de la Sierra: APCOB.

Combès, I. y D. Villar. (2007). Os Mestiços mais puros. Representações chiriguano e chané da mestiçagem. Mana, 13 (1), 41-62.

Córdoba, L. (2019). Una voz desde Sudamérica: sociedad, etnografía y religión en la obra de John Arnott. Boletín Americanista, 78, 91-111.

Daviet, W. (2016). Observations sociolinguistiques et analyse de la phonologie du dialecte ava du guaraní bolivien Langue tupi-guaraní de Bolivie. Tesis de Maestría, Université Lumière Lyon 2, París.

De Oré, L. G. (1607). Rituale, seu manuale Peruanum, et Forma Breuis Administrandi apud Indos sacrosancta Baptismi, Ponitentice, Eucharistice, Matrimonij, \& Extremce unctionis Sacramenta. Nápoles.

Dietrich, W. (1986). El idioma chiriguano: Gramática, textos, vocabulario, Ediciones Cultura Hispánica, Madrid.

Dietrich, W. (2007). Nuevos aspectos de la posición del conjunto Chiriguano (Guaraní del Chaco Boliviano) dentro de las lenguas Tupí-Guaraníes bolivianas. En A. RomeroFigueroa, A. Fernández Garay y Á. Corbera Mori, Lenguas indígenas de América del Sur. Caracas: Universidad Católica Andrés Bello, 9-18.

Dietrich, W. (2009). Sintaxis del guaraní chaqueño (chiriguano, tupí guaraní): La cláusula y las relaciones interclausales. Amerindia, 33-34, 333-363.

Dietrich, W. (2011). La función del sufijo guaraní -kue/-(n) gue. UniverSOS. Revista de lenguas indígenas y universos culturales, 8: 65-78. 
Dorais, L-J. (1970). L'acculturation lexicale chez les esquimaux du Labrador. Langages, 18, 65-77.

Estado Plurinacional de Bolivia. (2009). Constitución Política del Estado Plurinacional de Bolivia.

Giannecchini, D. (1869). Carta del P. Doroteo Giannecchini al R.P. guardián y venerable discretorio, 26.VI.1869. Tarija: Archivo Franciscano de Tarija [AFT 878].

Giannecchini, D. (1916 [1896]). Diccionario ChiriguanoEspañol y Español-Chiriguano. Tarija: Publicación de la Orden Franciscana.

Guasch, A. (1996 [1956]). El idioma guaraní. Gramática y antología de prosa y verso. Asunción: CEPAG.

Gustafson, B. (2014). Guaraní. En M. Crevels y P. Muysken. Lenguas de Bolivia. Tomo 3. Oriente (pp. 307-368). La Paz: Plural Editores.

Harwood, L. F. (1995). Ñaneñee Riru. Diccionario: Guaraní-Castellano. Charagua: Comité Hable Guaraní.

Harwood, L. F. (1996 [1995]). Ñaneñee Riru. Diccionario: Guaraní-Castellano. Charagua: Comité Hable Guaraní.

Harwood, L. F. (2003 [1995]). Ñaneñee Riru. Diccionario: Guaraní-Castellano. Camiri/Santa Cruz de la Sierra: Comité Hable Guaraní.

Haspelmath, M. (2008). Loanword typology: Steps toward a systematic cross-linguistic study of lexical borrowability. En T. Stolz, D. Bakker y R. Salas Palomo (Eds.), Aspects of language contact. (pp. 43-62). Berlín: Mouton de Gruyter.

Hirsch, S. (2003). The emergence of political organizations among the Guaraní Indians of Bolivia and Argentina: a comparative perspective. En: E. Langer y E. Muñoz, Contemporary Indigenous Movements in Latin America. (pp.81-101). Nueva York: Rowman \& Littlefield Publishers.

Hirsch S. y A. Alberico. (1996). El don de la palabra: un acercamiento al arte verbal de los guaraní de Bolivia y Argentina. Anthropos, 91 (1-3), 125-137.

Krivoshein De Canese, N. y F. Acosta Alcaraz. (2001). Gramática Guaraní. Asunción: Ñemity.

Langer, E. (2008). La experiencia chiriguana en la Guerra del Chaco y la destrucción de las misiones franciscanas. En N. Richard (Ed.), Mala guerra. Los indígenas en la guerra del Chaco (1932-1935) (pp. 235-250). París/Asunción: ServiLibro-Museo del Barro/CoLibris.

Langer, E. (2009). Expecting Pears from an Elm Tree: Franciscan Mission on the Chiriguano Frontier in the Heart of South America, 1830-1949. Durham/Londres:
Duke University Press.

León de Santiago, P. (1791). Diccionario Breve Chiriguanae. Tarija: Archivo Franciscano de Tarija [AFT-1-1835/MS16].

Matoré, G. (1953). La méthode en lexicologie: domaine français. París: Didier.

Métraux, A. (1930a). La sécularisation des Missions Franciscaines du Chaco Bolivien. Journal de la Société des Américanistes, 21 (2), 420-422.

Métraux, A. (1930b). Études sur la civilisation des indiens chiriguano. Tucumán: Universidad Nacional de Tucumán, Instituto de Etnología.

Métraux, A. (1948). Tribes of the Eastern Slopes of the Bolivian Andes. Chiriguano and Chané. En J. H. Steward (Ed.), Handbook of South American Indians, Vol. 3 (pp. 465-485). Washington: Smithsonian Institution.

Monino, Y. (1970). Dérivation, composition et emprunt dans le vocabulaire des techniques Ngbaka-Ma'bo (République Centrafricaine). La Linguistique, 6, 117-146.

Montani, R. (2017). Las verdaderas palabras de Dios en wichí. Sobre la terminología cristiana en las traducciones de los anglicanos. En: C. Ceriani Cernadas (Ed.), Los evangelios chaqueños: misiones y estrategias indígenas en el siglo XX (pp. 145-281). Buenos Aires: Rumbo Sur.

Morando, M. A. (2015). Bilingüismo y organización social en la comunidad chané de Tuyunti (departamento General San Martín, provincia de Salta). Suplemento Antropológico, 50 (2), 257-335.

Morando, M. A. (2017). Visiones de lo religioso entre los chiriguano: la lexicografía franciscana entre los siglos XVIII y XIX. Anuario de Estudios Bolivianos, Archivísticos y Bibliográficos, 23 (1), 199-221.

Morando, M. A. (2018). Producción misionera sobre la lingüística chiriguana: una mirada diacrónica. Anthropos, $113,151-167$.

Morando, M. A. (2020). Ñande ñee jekove: lengua y praxis social entre los chanés del noroeste argentino. Tesis de Doctorado, Universidad de Buenos Aires, Buenos Aires.

Nordenskiöld, E. (2002 [1912]). La vida de los indios. El Gran Chaco (Sudamérica). La Paz: APCOB/Plural.

Nordenskiöld, E. (1922). Deductions suggested by the geographical distribution of some post-Columbian words used by the Indians of S. America. Gotemburgo: Elanders Boktryckeri Aktiebolag. 
Nordlinger, R. y Sadler L. (2004). Nominal Tense in Crosslinguistic Perspective. Language, 80 (4), 776-806.

Ortiz García, E. y E. Caurey. (2011). Diccionario etimológico y etnográfico de la lengua guaraní hablada en Bolivia (guaraní-español). La Paz: SENAPI.

Otazú, A. (2006). Práctica y semántica en la evangelización de los Guaranies del Paraguay (S. SVI-XVIII). Asunción: CEPAG.

Paula Camargo, Jonathan de. (2020). Os neologismos como um processo de resistência: observações linguísticas na comunidade wakalitesu -Aldeia Três Jacus. Tesis de Maestría, Universidade Federal de Mato Grosso, Cuiabá.

Pitarch, P. y G. Orobitg. (2012). Modernidades indígenas. Madrid/Frankfurt: Iberoamericana/ Vervuert.

Riester, J. (2008). Iyambae- Ser Libre. La memoria de la Guerra del Chaco en la memoria indígena isoseña. En N. Richard (Ed.), Mala guerra. Los indígenas en la guerra del Chaco (1932-1935) (pp. 183-202). París/Asunción: ServiLibro-Museo del Barro/CoLibris.

Romero Yaguari, J. (2006). Gramática Elemental del Idioma Guaraní. Tarija: Gobernación del Departamento de Tarija.

Ruiz De Montoya, A. (1639). Tesoro de la lengua guaraní. Compuesto por el padre Antonio Ruiz, de la Compañia de lesus. Madrid: Juan Sánchez.

Ruiz De Montoya, A. (1640). Arte y vocabulario de la lengua guaraní. Compuesto por el padre Antonio Ruiz de la Compañia de lesus. Madrid: Juan Sánchez.

Schmidt, M. (1938). Los Chiriguanos e Izozós. Revista de la Sociedad Científica del Paraguay, 4 (3), 1-115.

Schuchard, B. (1979). Ñane ñë. Gramática guaraní para castellano-hablantes. Santa Cruz de la Sierra: APCOB.

Scionti, F. (2016). Pluralismo giuridico e pratica giuridica indigena. Il caso dei Guaranì del Chaco Boliviano. EtnoAntropologia, 4 (1), 111-130.

Susnik, B. (1968). Chiriguanos I. Dimensiones etnosociales. Asunción: Museo Etnográfico Andrés Barbero.

Thévet, A. (1586). Le grand insulaire, et pilotage d'André Thevet. Angoumoisin, cosmographe du Roy, dans lequel sont contenus plusieurs plants d'isles habitées, et deshabitée, et description d'icelles. París.

Veliz, J.D. (1999). Nemoesakaa. La Paz: UNICEF.

Villar, D. (2006). Repensando el complejo cultural chiriguano-chané. En I. Combès (Ed.), Definiciones étnicas, organización social y estrategias políticas en el Chaco y la Chiquitanía (pp. 205-224). Santa Cruz de la Sierra/Lima: Institut Français d'Études Andines.

Villar, D. (2008). Guaranización, traducción y evangelización en las representaciones anímicas chané. Suplemento Antropológico, 43 (1), 339-386.

Zajícová, L. (2009). Variación estilística en el contacto lingüístico: el caso del guaraní y el español en Paraguay. Études Romanes de Brno 30 (2): 203-211.

Zimmermann, K. (2006). El problema del purismo en la modernización de las lenguas amerindias. En R. Terborg y L. G. Landa (Comps.), Los retos de la planificación del lenguaje en el siglo XXI. México: Universidad Nacional de México. 